\title{
INTERACTION METRICS FOR FEEDBACK CONTROL OF SOUND RADIATION FROM STIFFENED PANELS
}

\author{
Randolph H. Cabell; David E. Cox† Gary P. Gibbs* \\ NASA Langley Research Center, Hampton, VA 23681
}

\begin{abstract}
Interaction metrics developed for the process control industry are used to evaluate decentralized control of sound radiation from bays on an aircraft fuselage. The metrics are applied to experimentally measured frequency response data from a model of an aircraft fuselage. The purpose is to understand how coupling between multiple bays of the fuselage can destabilize or limit the performance of a decentralized active noise control system. The metrics quantitatively verify observations from a previous experiment, in which decentralized controllers performed worse than centralized controllers. The metrics do not appear to be useful for explaining control spillover which was observed in a previous experiment.
\end{abstract}

\section{Introduction}

A CTIVE control of sound radiation due to turbulent boundary layer excitation of an aircraft fuselage has been the subject of much recent research. This problem is typically treated as one of minimizing sound radiation from individual bays of the aircraft fuselage, where a bay is a rectangular portion of the fuselage skin bounded by ring frames and stringers. The random, distributed nature of turbulent boundary layer (TBL) excitation means neighboring bays are driven by a spatially and temporally uncorrelated forcing function.

The most common active control approaches assume feedback of a structural sensor such as an accelerometer or piezoceramic transducer. ${ }^{1-4}$ Feedforward control is less common in this application due to the absence of a reference signal which is correlated with the TBL excitation. Numerous analytical studies have demonstrated the potential for feedback control on this problem. ${ }^{3,5-7}$ The feasibility of the approach has also been demonstrated in various experiments. ${ }^{1,8-10}$

Although active control is promising on individual fuselage bays, significant implementation issues must be addressed before a system can be deployed on an aircraft containing several hundred bays. For example, the control system components should be as simple and reliable as possible. Equally important is the question of a centralized versus a decentralized control scheme. For this application, the question is whether feedback control is implemented using only local information, or if a centralized controller coordinates multiple feedback loops. A decentralized control system which relies only on local information is generally preferred for reasons of simplicity and fault tolerance. ${ }^{11}$

\footnotetext{
*Structural Acoustics Branch

${ }^{\dagger}$ Guidance and Control Branch
}

Much of the recent research in controlling sound radiation from panels has implicitly addressed this question by studying control systems which use matched, collocated actuator and sensor pairs. ${ }^{2,3,12}$ A matched, collocated actuator and sensor pair is physically collocated on the structure and has the same function form for the modal coupling coefficients. ${ }^{13}$ This arrangement can be guaranteed to be dissipative, or passive, meaning it is inherently stable and tolerant to changes in the system being controlled. A matched, collocated controller is suitable for decentralized control because there is no need to account for destabilizing inputs from neighboring controllers. In spite of these benefits, this approach is categorized as a low-authority approach, since in practice passivity often severely limits the performance achievable through active control.

Higher authority approaches based on linear quadratic optimal or robust control designs have also been studied for this problem. ${ }^{1,8,10}$ These approaches lack the robustness and stability guarantees of a matched, collocated controller, and are more complicated, but generally have better performance. ${ }^{3}$ If decentralized control is to be implemented with a higher authority approach, the design of the feedback loops may have to consider the destabilizing effect of inputs from neighboring controllers. For example, in a recent experiment comparing centralized and decentralized controllers on an aircraft-style sidewall, noise reduction performance was limited by stability issues in the implementation of decentralized designs. ${ }^{9}$

The present paper considers the design and implementation of decentralized controllers on an aircraft sidewall when the control system does not involve a matched actuator/sensor pair. Experimentally measured frequency response functions are examined using interaction metrics which have been previously described in the process control literature. ${ }^{11,14}$ These 
interaction metrics highlight frequencies where decentralized controllers could destabilize one another due to coupling in the system being controlled. The destabilizing effects could be mitigated either in the design of the individual decentralized control loops, or by implementing some degree of centralization, or coordination, among multiple feedback controllers.

Because of the complex dynamics of a built-up structure such as an aircraft fuselage, the analysis here uses data measured from a physical model of a fuselage sidewall. This model was constructed in order to study the control of sound radiation due to TBL excitation of an aircraft fuselage. This ensures the data has some of the complexities of an actual aircraft fuselage, such as varying boundary conditions due to manufacturing variabilities. But, it also means the analysis will include dynamics which may be particular to this specific structure. Nonetheless, the analysis is useful for illustrating the interactions and control design considerations which may be found on a real fuselage.

The paper begins with a description of a decentralized control system, and suggests two metrics for analyzing interactions between feedback controllers. The experimental setup, which consists of a fuselage-style panel in a wind tunnel, is described next, including the data which were measured as part of the previous test. ${ }^{9}$ The interaction metrics are then applied to this data and compared with the known panel dynamics in order to highlight areas where inter-bay coupling could present problems for a decentralized controller. The paper closes with a discussion of closed loop data for two control designs which had substantial spillover, possibly due to unmodeled interactions between neighboring feedback loops.

\section{Analysis}

This section introduces notation useful for analyzing the decentralized control problem and discusses two interaction metrics. Decentralized control is a common topic in controls textbooks, but interaction metrics described in these textbooks have not previously been applied to measured data from an aircraft-style structure. This section summarizes the details needed to understand the metrics, and closely follows the excellent discussion of decentralized control given in Chapter 14 of Morari and Zafiriou. ${ }^{14}$

A block diagram of a decentralized control system is shown in Fig. 1. The $G(z)$ block denotes a plant with $m$ inputs and $m$ outputs, where $u_{i}$ denotes the $i$ th input and $y_{i}$ denotes the $i$ th output. Feedback controllers are designated by the $k_{i}(z)$ blocks, where the $i$ th controller receives input from only the $i$ th plant output and drives only the $i$ th plant input, as shown in the figure.

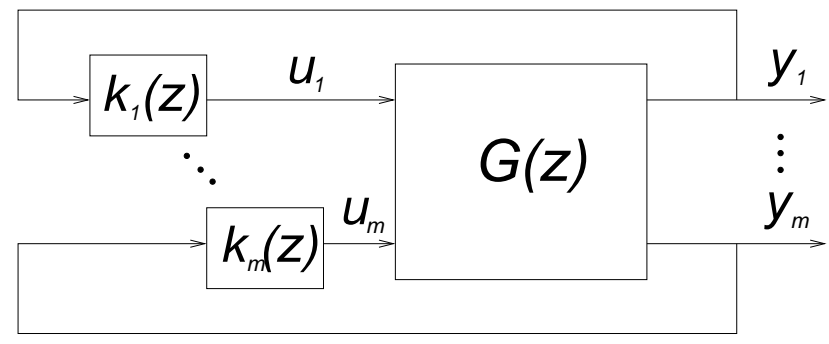

Fig. 1 Decentralized Control of plant $G(z)$

Given this decentralized control topology, an important question is the extent to which interactions between neighboring actuators and sensors will destabilize or reduce the performance of the control system. At the simplest level, this question concerns the magnitude of off-diagonal terms in the plant, $G$, relative to the diagonal terms. For example, if the transfer function from the $i$ th actuator to the $i$ th sensor is denoted $g_{i i}$, then the off-diagonal term from the $j$ th actuator to the $i$ th sensor would be denoted $g_{j i}$. If the off-diagonal terms are large relative to the diagonal terms, it is easy to envision a scenario where decentralized control loops could fight one another and eventually go unstable.

A decentralized control scheme allows for lower order models, simpler control design techniques and has a significant advantage in implementation as there is no need to provide for signal paths between adjacent panel bays. Hence the goal is to design and implement the individual feedback loops as if the plant $G$ were diagonal. Interaction metrics can help understand how coupling, due to off-diagonal elements of $G$, constrains the design of the decentralized feedback loops. ${ }^{11,14}$ Significant coupling could constrain the loops to be very weak controllers in order to preserve stability of the fully coupled system. Although some insight into this question is available by examining the cross terms of a frequency response function, this quickly becomes unwieldy for multiple feedback loops and does not yield direct design guidelines.

\section{Relative gain array metric}

This leads to the first interaction metric to be discussed, which is the relative gain array (RGA). RGA can be used to quantify the diagonal dominance of a plant such as $G$ in Fig. 1. The RGA is defined as ${ }^{14}$

$$
\Lambda(G(\omega))=G(\omega) \times\left(G(\omega)^{-1}\right)^{T}
$$

where $\times$ denotes the element by element product. Thus the RGA of a frequency dependent square matrix $G$ is the same size as $G$ and is also frequency dependent. A few properties of the RGA which are relevant for analyzing a decentralized controller are:

- $\Lambda(G)$ is independent of scaling on the inputs and 
outputs of $G$

- $\Lambda(G)$ is close to the identity matrix for plants which are diagonally dominant. The RGA number, or sum norm of the RGA matrix, quantifies the closeness of the RGA to the identity matrix: ${ }^{11}$

$$
\text { RGA number }=\|\Lambda-I\|_{\text {sum }}
$$

- if $G$ is stable and each decentralized controller is stable, then $\Lambda(G)=I$ implies stability of the entire system. ${ }^{11}$

When the plant is diagonally dominant, it is unlikely that the operation of one decentralized controller will have any impact on the operation of any other controller, which is obviously desirable. The RGA has a simple form for a $(2 \times 2)$ system:

$$
\Lambda=\left[\begin{array}{cc}
\lambda_{11} & 1-\lambda_{11} \\
1-\lambda_{11} & \lambda_{11}
\end{array}\right]
$$

where $\lambda_{11}=g_{11} g_{22} /\left(g_{11} g_{22}-g_{12} g_{21}\right)$.

Note that the RGA can be computed from a frequency response function description of a plant. This means some insight into the diagonal dominance of a plant can be obtained simply by examining measured input/output data. There is no need to compute a realization (e.g. a state-space or ARMA model) of the plant under consideration. Because the RGA is scaling independent, a simple test for diagonal dominance is to look for elements of $\Lambda(G)$ which are much greater than one, or equivalently where $\Lambda(G)$ is significantly different from the identity matrix.

The RGA is most commonly discussed in the context of the pairing problem, where one must decide which inputs to pair with which outputs. ${ }^{11,14}$ It is useful in that context because it only needs to be computed once for the candidate input/output set, and different actuator/sensor pairings can be studied without having to recompute the RGA. ${ }^{11}$ Here, however, the desire to have independent control loops on each fuselage bay uniquely determines the actuator/sensor pairing.

Although the RGA is useful for analyzing the diagonal dominance of a plant, it does not provide bounds on the allowable control gains.

\section{$\mu$ metric}

A more detailed interaction metric which does provide gain bounds can be derived using concepts from robust control analysis. Following the approach described in Morari and Zafiriou, ${ }^{14}$ the block diagram of the decentralized control system can be redrawn as shown in Fig. 2. This diagram is equivalent to the first diagram, but it illustrates how the off-diagonal elements of $G$ can be viewed as perturbations on the

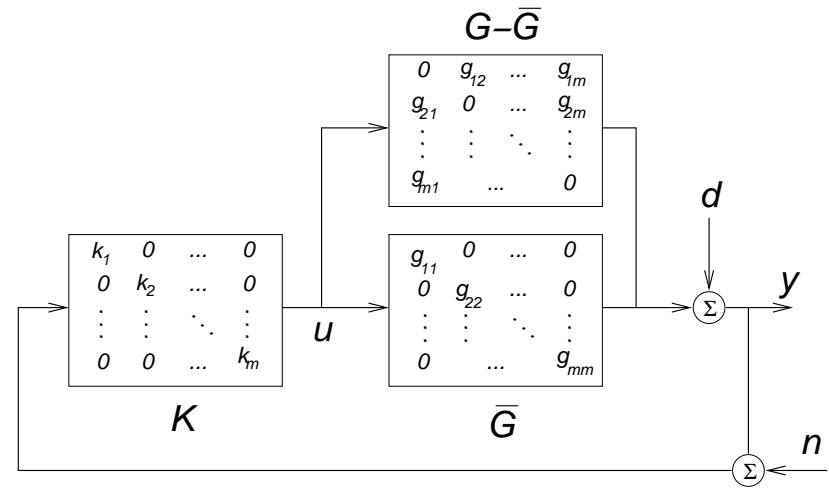

Fig. 2 Decentralized control schematic for interaction analysis

diagonal elements. The control loops, $k_{i}$, are designed for the diagonal plant, $\bar{G}$. The stability of the resulting decentralized controller, $K$, on the full system, $G$, can then be quantified by the robustness of the controller with respect to the additive uncertainty, $(G-\bar{G})$. The additional signal, $d$, in the figure corresponds to a disturbance input measured by the sensors, and $n$ denotes random sensor noise.

At this point it is helpful to introduce a few standard quantities for analyzing the control system. The sensitivity of the system in the figure is defined as a transfer function from $d$ to $y,{ }^{15}$ and is written

$$
E=\frac{I}{I-G K}
$$

Similarly, the complementary sensitivity, which is the transfer function from $n$ to $y$, is written

$$
H=\frac{G K}{I-G K}
$$

where the relationship

$$
E+H=I
$$

holds. ${ }^{15}$ These quantities have been defined assuming positive feedback in order to be consistent with the experimental results discussed later in the paper.

Some general, well-known ${ }^{15}$ properties of these transfer functions are worth mentioning. For good suppression of the disturbance, $d$, the sensitivity $E$, should be small where $d$ is significant. For a MIMO control system, this requirement can be written in terms of the maximum singular value of $E$, denoted $\bar{\sigma}(E)$, as

$$
\bar{\sigma}(E) \ll 1
$$

Similarly, to minimize the effect of the noise $n$ on the response $y$, the complementary sensitivity $H$ should be small: $\bar{\sigma}(H) \ll 1$. 
The return difference for the system in Fig. 2 can be factored as ${ }^{11,14}$

$$
(I-G K)=\left(I-L_{H} \bar{H}\right)(I-\bar{G} K)
$$

where

$$
L_{H}=(G-\bar{G}) \bar{G}^{-1}
$$

quantifies the relative error in approximating $G$ by the diagonal $\bar{G}$. The second term on the right hand side of Eq. 8 is the return difference if the plant is assumed to be diagonal (i.e. $G=\bar{G}$ ). The complementary sensitivity for the diagonal plant is written

$$
\bar{H}=\frac{\bar{G} K}{I-\bar{G} K}
$$

which is itself diagonal. The $i$ th diagonal of $\bar{H}$ can be written

$$
h_{i}=\frac{g_{i i} k_{i}}{1-g_{i i} k_{i}}
$$

The full $\bar{H}$ is stable if each loop, $h_{i}$, is stable. If $\bar{H}$ is stable, then the full control system is stable if and only if the Nyquist criterion is satisfied for the first term on the right hand side of Eq. 8: $\left(I-L_{H} \bar{H}\right) .{ }^{14}$

From the small gain theorem, a sufficient condition for stability is to limit the spectral radius of $L_{H} \bar{H}$, denoted $\rho\left(L_{H} \bar{H}\right)$, such that

$$
\rho\left(L_{H} \bar{H}\right)<1
$$

This is satisfied if the product of induced norms is less than unity,

$$
\left\|L_{H}\right\|\|\bar{H}\|<1
$$

This equation provides a simple measure for design purposes. $L_{H}$ is strictly a function of the open loop plant so it is independent of controller design. Furthermore $L_{H}$ can be computed from experimental frequency response data without requiring a system realization. If $\bar{H}$ is diagonal, $\|\bar{H}\|=\max \left|h_{i}\right|$, provides a gain bound for the controller that need only consider the most aggressive loop.

However, the simplicity of the metric in Eq. 13 comes at a price of conservativeness. The norm condition in Eq. 13 is only sufficient for the Nyquist criterion, and therefore can be quite conservative. One way to reduce this conservatism is by applying a frequency dependent scaling to the norm calculation on the normalized plant error, $L_{H}$. This yields a structured singular value, ${ }^{16,17}$ or $\mu$, condition for stability as $^{14}$

$$
\|\bar{H}(\omega)\|<\mu^{-1}\left(L_{H}(\omega)\right), \quad 0 \leq \omega \leq \pi / T
$$

where $\mu(\cdot)$ yields a real, positive scalar. $\mu$ is computed for a particular uncertainty structure, which for decentralized control corresponds to the diagonal structure of $\bar{H}$. The use of Eq. 14 in place of Eq. 13 can reduce conservatism considerably.

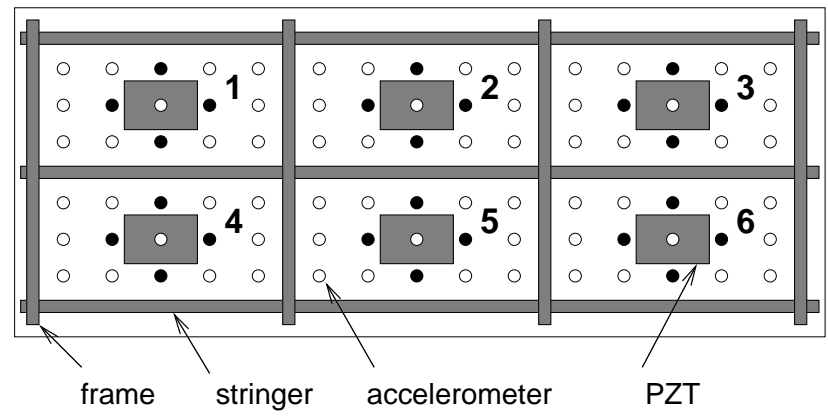

Fig. 3 Test panel schematic

\section{Experimental Setup}

The interaction metrics were applied to data collected in a previous controls experiment. ${ }^{9}$ This interaction analysis was done by re-examining frequency response and closed loop data from the earlier experiment.

\section{Test Panel}

The test configuration has been described in detail elsewhere ${ }^{1,9}$ and is summarized here. The test structure consists of an aluminum panel which models the sidewall of an aircraft fuselage, absent the fuselage curvature. A schematic of the panel is shown in Fig. 3. The structure consists of six bays separated by vertical aluminum frames and horizontal stringers. The dimensions of each bay are $50.8 \mathrm{~cm} \times 25.4 \mathrm{~cm} \times 0.16 \mathrm{~cm}$. The bays are referred to in later sections of the paper by the numbers indicated on the schematic. In-plane tension was applied to the panel in the vertical direction to simulate the hoop stress in a pressurized aircraft flying at $12,000 \mathrm{~m}$.

The panel was mounted in the sidewall of the Structural Acoustic Flow Apparatus (SAFA) at NASA Langley Research Center. SAFA is a low speed wind tunnel which is used to study turbulent boundary layer excitation of panels. A photo of the panel installed in the tunnel sidewall is shown in Fig. 4.

\section{Transducers}

The control system on each bay consisted of a single actuator and four accelerometers whose outputs were summed and treated as a single sensor. The actuator was a center-mounted piezoceramic (PZT-5A, $15.2 \mathrm{~cm} \times 10.2 \mathrm{~cm} \times 0.038 \mathrm{~cm})$. The sensor response on each bay was created by summing the response of four accelerometers which were arranged in a diamond pattern centered on the PZT. The four accelerometers in the diamond array on each bay are indicated in the schematic by blackened circles. The output of this sensing configuration is an approximate measure of the response of the first radiation mode of the panel. ${ }^{1}$ Eleven additional accelerometers were 


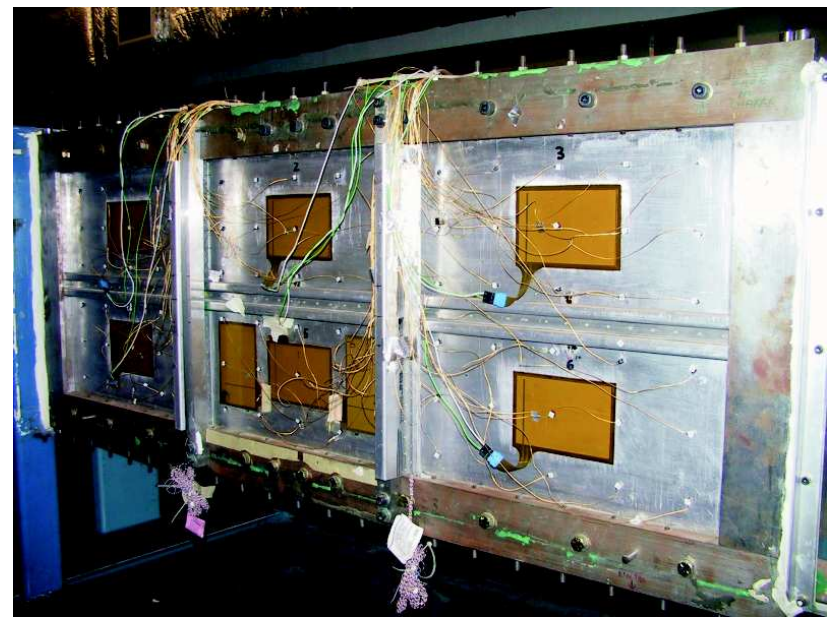

Fig. 4 Test panel mounted in wind tunnel

mounted on each bay and used in a separate data acquisition system to estimate the radiated sound power from each bay.

The decentralized control topology considered here consisted of independent single input/single output (SISO) control loops running on each bay. The centralized control topology consisted of a fully coupled, multiple-input/multiple-output (MIMO) topology, where the number of inputs and outputs equaled the number of bays being controlled.

Frequency response data between actuators and sensors were collected using the digital control system. This ensured an accurate measurement of all gains and delays in the control path. The sample rate for all data was $3000 \mathrm{~Hz}$.

\section{Results and Discussion}

The interaction metrics were used to analyze decentralized control of two pairs of bays on the test panel. The first pair consisted of bays one and two, and the second pair consisted of bays one and four. A more realistic interaction analysis will have to include more than just two bays at a time, especially if decentralized control is to be implemented on an aircraft fuselage. However, only two bays at a time are considered here to simplify interpretation of the results.

The full plant matrix, $G$, for each two-bay configuration was either

$$
\left[\begin{array}{ll}
g_{11} & g_{12} \\
g_{21} & g_{22}
\end{array}\right] \quad \text { or } \quad\left[\begin{array}{ll}
g_{11} & g_{14} \\
g_{41} & g_{44}
\end{array}\right]
$$

depending on which pair of bays was being studied. One obvious centralized controller for this system is a two input/two output (or MIMO) configuration which considers off-diagonal terms of $G$ in the control design. A decentralized configuration consists of two single input/single output (SISO) controllers which neglect off-diagonal terms in the design of the individual SISO control loops.

Experimentally measured values of the matrices in Eq. 15 as a function of frequency are shown in Fig. 5. The figures show measured frequency response functions (FRFs) in terms of average acceleration, across the diamond array, per unit voltage input to the PZT. The data on bays 1 and 2, in Figs. 5(a) and 5(b), were collected by simultaneously driving the PZTs on those bays with independent random noise sequences. Similarly, the data on bays 1 and 4, in Figs. 5(c) and 5(d), were collected by simultaneously driving the PZTs on bays 1 and 4 only. In each plot, the solid line shows the FRF from the PZT to the sensor on the same bay, while the dashed line shows the sensor response on the neighboring bay. The frequency range in the plots is restricted to $150 \mathrm{~Hz}$ to $700 \mathrm{~Hz}$ for simplicity. Coupling occurs outside this frequency range, but the selected range is of particular interest for active control.

A simple check for diagonal dominance of each $(2 \times 2)$ plant is to examine the separation between the solid and dashed lines in Fig. 5. Frequencies where the two lines coincide, or where the dashed line lies above the solid line, indicate regions where the plant is not diagonally dominant. Several such areas are visible in the plots. For example, the solid and dashed lines coincide near $180 \mathrm{~Hz}$ in all four plots. For bays 1 and 2, Figs. 5(a) and 5(b) show coupling near $325 \mathrm{~Hz}, 425 \mathrm{~Hz}$, and $625 \mathrm{~Hz}$. On bays 1 and 4, coupling is evident near $260 \mathrm{~Hz}, 325 \mathrm{~Hz}, 375 \mathrm{~Hz}$, and $425 \mathrm{~Hz}$.

The operating deflection shapes offer some insight into the panel dynamics at these frequencies. The deflection shapes at four frequencies of interest, due to a random input to the PZT on bay one, are shown in Fig. 6. These were obtained by plotting the real part of the phased responses of the fifteen accelerometers on the panel. They reveal that many of the FRF peaks correspond to resonances of multiple, coupled bays. For example, $180 \mathrm{~Hz}$, in Fig. 6(a), corresponds to be a $(3,1)$ entire panel mode, with a nodal line on each of the vertical panel frames. Because it is a panel mode, there is significant coupling between all bays on the panel. At $260 \mathrm{~Hz}$, the driven bay and its vertical neighbor each vibrate in their $(1,1)$ bay modes, but $180^{\circ}$ out of phase with one another. There is considerably less coupling between the side by side bays at this frequency. For example, the response of bay 2 when bay 1 is driven (Fig. 5(a)) is considerably lower than the response of bay 4 when bay 1 is driven (Fig. 5(c)).

Coupling between side by side bays is present above $600 \mathrm{~Hz}$. The peak in Figs. 5(a) and 5(b) at $625 \mathrm{~Hz}$ corresponds to the resonance of the $(5,1)$ bay mode of bay one. The peak at $641 \mathrm{~Hz}$ in those figures is the $(5,1)$ bay mode of bay two. The FRF data shows significant 


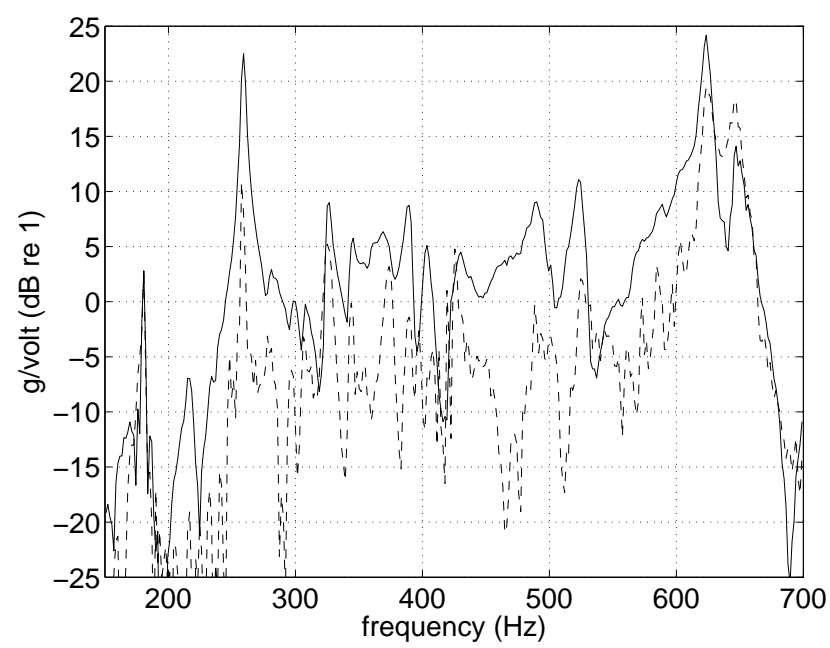

a) $g_{11}$ (solid), $g_{12}$ (dashed).

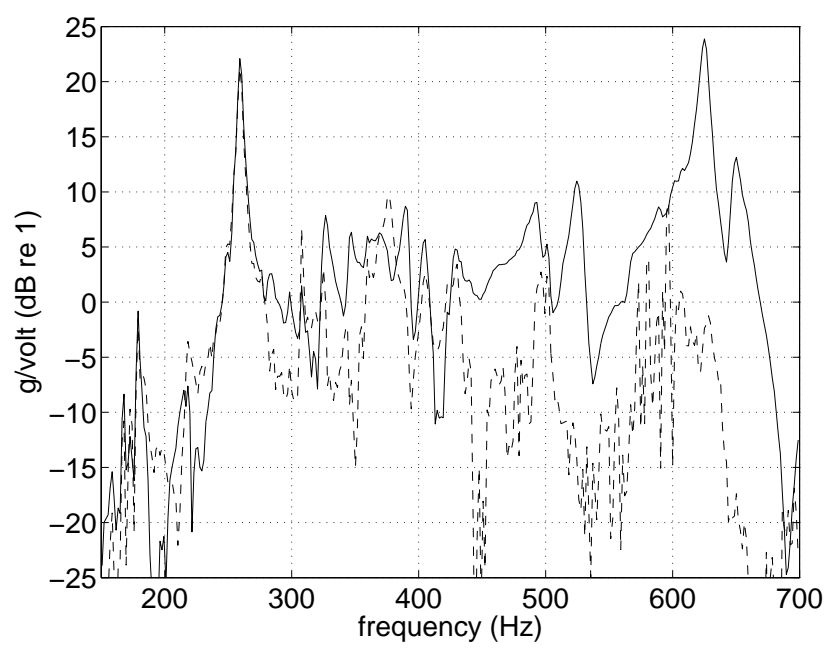

c) $g_{11}$ (solid), $g_{14}$ (dashed).

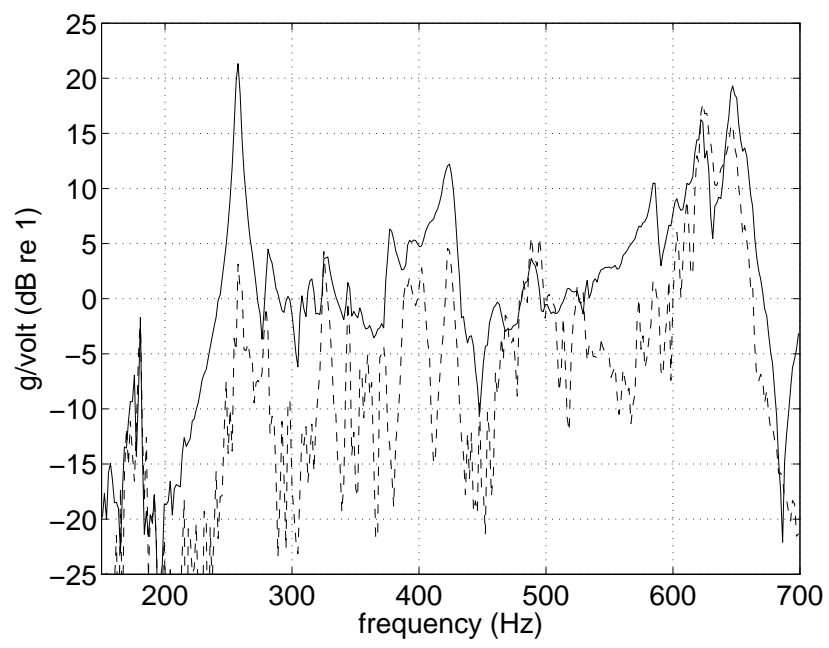

b) $g_{22}$ (solid), $g_{21}$ (dashed).

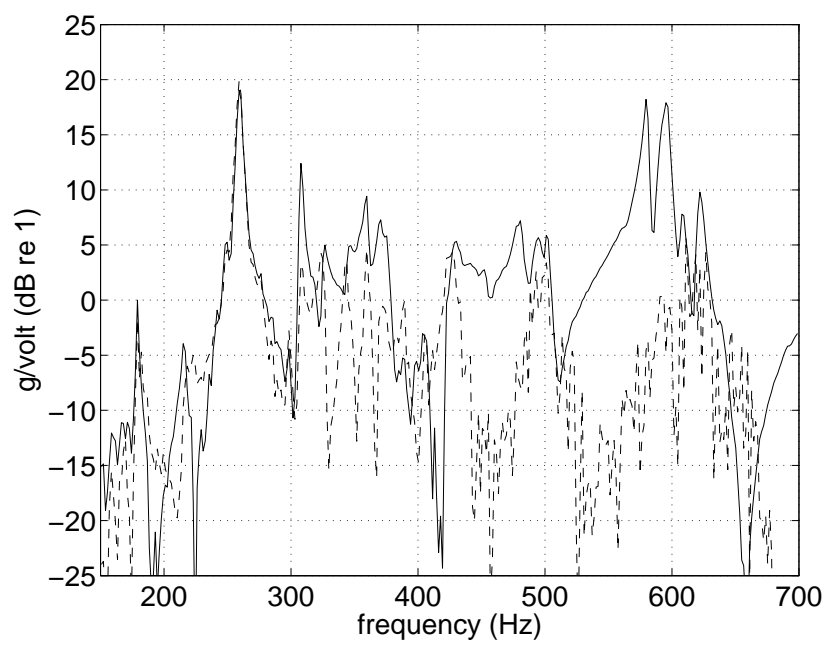

d) $g_{44}$ (solid), $g_{14}$ (dashed).

Fig. 5 Frequency responses from measured data: (a), (b) bays 1 and 2; (c), (d) bays 1 and 4

coupling between these bays in this frequency range.

Although the FRFs are informative, the interaction metrics offer more concise information on bay interactions. The RGA number from Eq. 2 is shown in Fig. 7 for bays one and two. Wherever the RGA number is non-zero, the RGA matrix differs from the ideal identity matrix and the plant $G$ is not diagonally dominant. The plot shows that for much of the frequency range, the diagonal terms of $G$ are dominant. The only areas where the number deviates significantly from zero are near the panel mode at $180 \mathrm{~Hz}$ and above $600 \mathrm{~Hz}$. The coupling near $180 \mathrm{~Hz}$ could be problematic for active control of sound radiation. Low order panel modes, such as at $180 \mathrm{~Hz}$, are more efficient sound radiators than higher order panel modes. If the authority of the decentralized controllers have to be reduced here to maintain stability, noise reduc- tion performance could be impacted. The coupling near $600 \mathrm{~Hz}$ may be less of a problem because it is associated with a higher order mode. The gain of the controllers could be rolled of above $600 \mathrm{~Hz}$ to avoid the possible destabilizing affects of this coupling.

The RGA number for bays one and four is shown in Fig. 8. The data indicates the plant matrix is not diagonally dominant below around $450 \mathrm{~Hz}$. In addition to the peak near $180 \mathrm{~Hz}$, there are several large peaks between 240 and $275 \mathrm{~Hz}$. These peaks generally agree with the FRF data, which suggested significant coupling between these bays in this frequency range. This coupling between the $(1,1)$ bay modes at $260 \mathrm{~Hz}$ can also present a problem for decentralized control of sound radiation, as with the $180 \mathrm{~Hz}$ mode. The RGA number is close to zero above $600 \mathrm{~Hz}$, which is expected since the FRFs indicated very little two way 


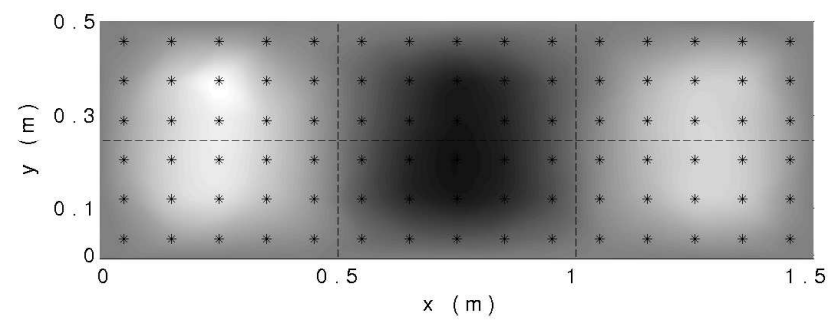

a) $180 \mathrm{~Hz}$



c) $625 \mathrm{~Hz}$

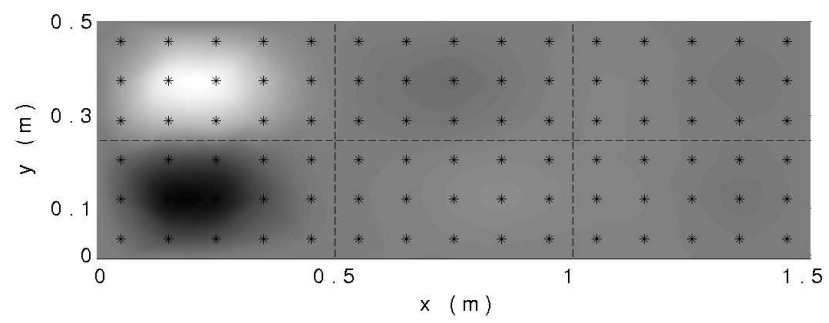

b) $260 \mathrm{~Hz}$

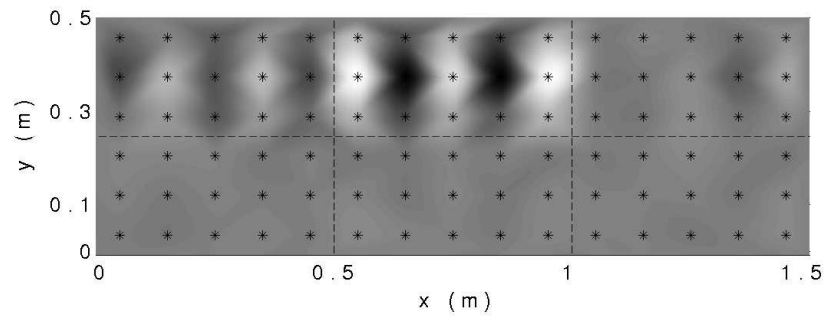

d) $641 \mathrm{~Hz}$

Fig. 6 Operating deflection shapes due to input on bay one.

coupling between these bays in this frequency range.

To summarize the RGA data for these bay pairs: the plant $(G)$ for bays one and two is diagonally dominant across a large frequency range except near the panel mode at $180 \mathrm{~Hz}$ and above $600 \mathrm{~Hz}$. In contrast, the plant for bays one and four is diagonally dominant only for frequencies above about $450 \mathrm{~Hz}$. If decentralized control is to be used on either bay pair, the possible destabilizing effects of these areas where the plants are not diagonally dominant should be considered. This appears to be most important for bays one and four.

The interaction metric $\mu\left(L_{H}\right)$ is plotted for both bay pairs in Fig. 9. The solid line corresponds to bays 1 and 2, while the dashed line corresponds to bays 1 and 4. As shown in Eq. 14, $\mu\left(L_{H}\right)$ provides a numeric constraint on the diagonal complementary sensitivity, $\bar{H}$.

For bays one and two the $\mu$ data generally agrees with the RGA data. $\mu\left(L_{H}\right)$ for these bays is greater than unity near $180 \mathrm{~Hz}$ and above $620 \mathrm{~Hz}$. For bays one and four, $\mu\left(L_{H}\right)$ indicates some additional frequency regions where the plant may not be diagonally dominant. For example, $\mu\left(L_{H}\right)$ peaks near $225 \mathrm{~Hz}$ but only slightly exceeds unity at $260 \mathrm{~Hz}$. In contrast, the RGA peaked at $260 \mathrm{~Hz}$, and was unity at $225 \mathrm{~Hz} . \mu\left(L_{H}\right)$ also exceeds unity at $320 \mathrm{~Hz}$ and $380 \mathrm{~Hz}$, and then peaks at $420 \mathrm{~Hz}$.

To summarize, the $\mu\left(L_{H}\right)$ metric confirms that stable decentralized control of bays one and two can be achieved with relatively little constraint on $\bar{H}$. For bays one and four, however, $\mu\left(L_{H}\right)$ indicates several frequency ranges where $\bar{H}$ is constrained, if stability of the decentralized controller is to be guaranteed.

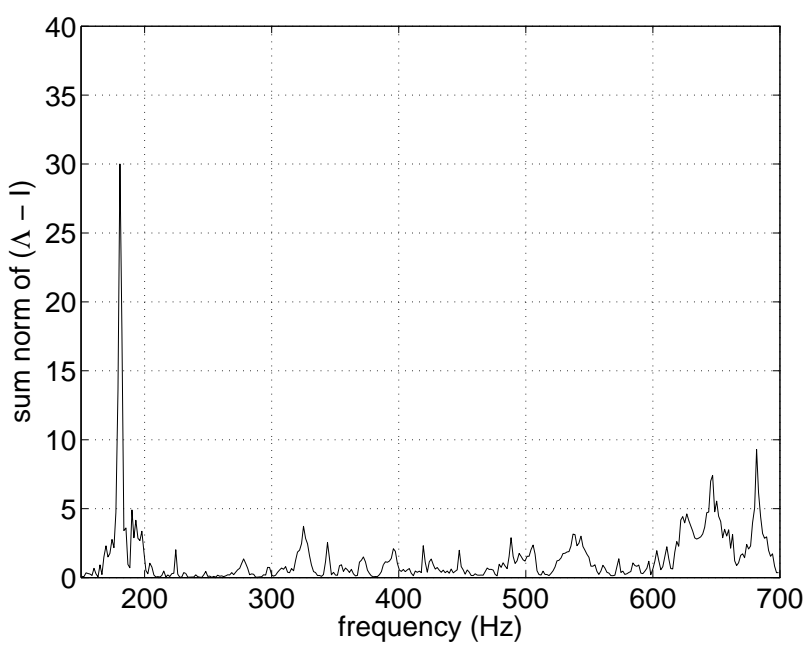

Fig. 7 RGA number for bays 1 and 2

\section{Analysis of Closed Loop Data}

Two decentralized control designs from an earlier experiment are reexamined here. The first controller was designed for bays one and two on the panel, and the second controller was designed for bays one and four. In both control designs the off-diagonal terms of the plant were ignored. These particular controllers were chosen because they were stable but had substantial spillover of control energy. The closed loop results and the metric in Eq. 14 are compared in order to evaluate the metrics for this application.

Before discussing the controllers in detail, typical results for centralized and decentralized control of these bay pairs are summarized here. The results are listed in Table 1 in terms of the change in radiated sound 


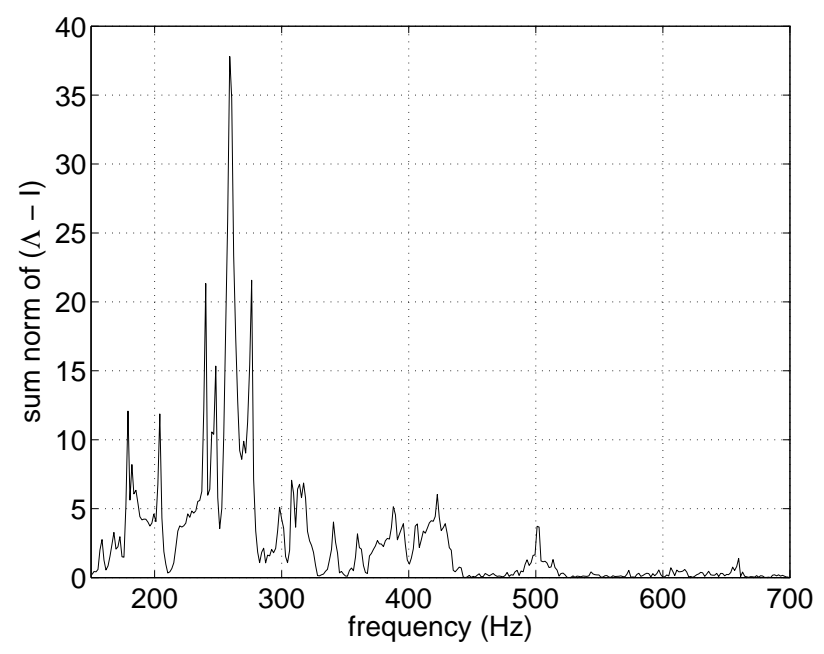

Fig. 8 RGA number for bays 1 and 4

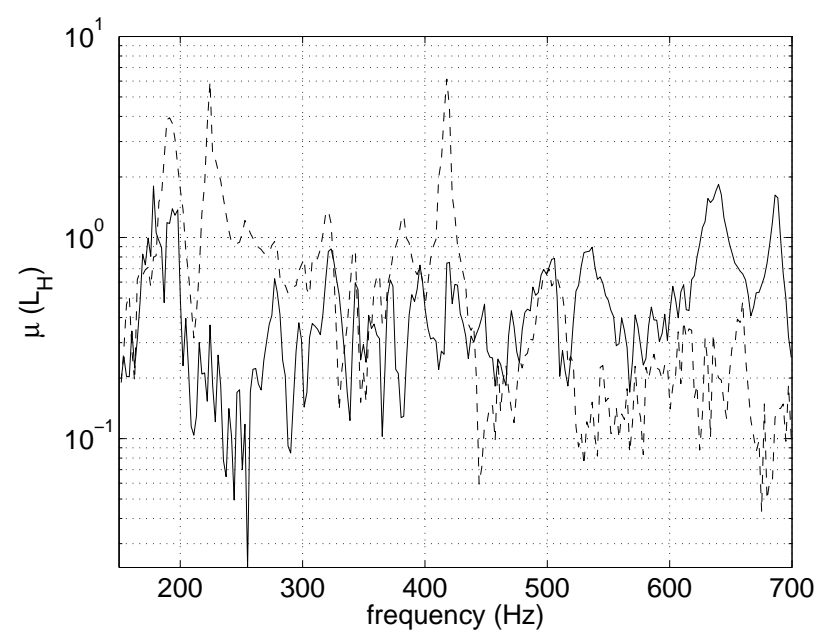

Fig. $9 \mu\left(L_{H}\right)$ for bays 1 and 2 (solid line); bays 1 and 4 (dashed line)

power with control relative to the no control case, on each bay of interest. The reductions were computed from the fifteen accelerometer responses, and were integrated from 150 to $800 \mathrm{~Hz}$. The corresponding tunnel flow speed was Mach 0.125. The first column specifies the control type, either centralized (MIMO) or decentralized (SISO). The next two columns show sound power reductions measured from bays 1 and 2 when those two bays were controlled. The last two columns show sound power reductions when only bays 1 and 4 were controlled. The results demonstrate that for both bay pairs, the modest reductions obtained with a centralized design could not be obtained with the decentralized designs. As discussed by Gibbs, ${ }^{9}$ the decentralized designs would go unstable before achieving the reduction performance of the centralized designs.

Both centralized and decentralized control designs

\begin{tabular}{|c|cc||cc|}
\hline & \multicolumn{4}{|c|}{ Bay Number } \\
\hline Control & 1 & 2 & 1 & 4 \\
\hline MIMO & $-3.0 \mathrm{~dB}$ & $-5.4 \mathrm{~dB}$ & $-3.0 \mathrm{~dB}$ & $-2.7 \mathrm{~dB}$ \\
\hline SISO & $-2.2 \mathrm{~dB}$ & $-3.1 \mathrm{~dB}$ & $-1.4 \mathrm{~dB}$ & $-1.4 \mathrm{~dB}$ \\
\hline
\end{tabular}

Table 1 MIMO vs. SISO control results ${ }^{9}$

were computed using a predictive control formulation, as described previously. ${ }^{9}$ The controllers were computed to minimize a cost function of the form

$$
J=\sum_{k=0}^{p-1}\|y(k)\|^{2}+\lambda\|u(k)\|^{2}
$$

The parameter $p$ is the cost horizon over which the control solution is optimal. ${ }^{18,19}$ The solution approximates a linear quadratic regulator for large values of $p$. A state estimator is required when implementing the controller, so the resulting control implementation is very similar to an LQG control solution. ${ }^{15}$ No frequency shaping of either the response or control effort terms in Eq. 16 was used. For the SISO (decentralized) control designs, this means that possible destabilizing effects of coupling were neglected.

A high order state space model of the plant was computed using an observer Kalman filter realization algorithm. ${ }^{20}$ For both MIMO and SISO control designs, the control effort parameter, $\lambda$, in Eq. 16 was reduced to the smallest value that still produced a stable controller when implemented. This approach may not produce controllers which are robust to plant variations, but it provided a consistent design goal for the different control configurations.

Auto-spectra of the sensor response on bay one with and without control are shown in Fig. 10 for decentralized control of bays one and two. The sensor is the summed response of the diamond accelerometer array. The solid line shows the auto-spectrum without control, and the dashed line shows the response with control. The open loop response is dominated by the panel mode at $180 \mathrm{~Hz}$, but this was reduced by $10 \mathrm{~dB}$ by the control system. Spillover is evident at $300 \mathrm{~Hz}$, 350 to $380 \mathrm{~Hz}$, and between 400 and $500 \mathrm{~Hz}$.

Although the RGA and the $\mu\left(L_{H}\right)$ metric indicated potential coupling problems at $180 \mathrm{~Hz}$ and above $600 \mathrm{~Hz}$, the control system performed fine at these frequencies. To understand this, the more detailed metric in Eq. 14 was computed and is shown in Fig. 11 for this decentralized control configuration. The product of $\bar{\sigma}(\bar{H})$ and $\mu\left(L_{H}\right)$ should be less than unity to guarantee stability of the decentralized controller. The data in the figure show that this metric was satisfied except near $450 \mathrm{~Hz}$ and near $640 \mathrm{~Hz}$. Thus even though the control design did not consider the constraint imposed by $\mu\left(L_{H}\right)$, the resulting design happened to satisfy the 


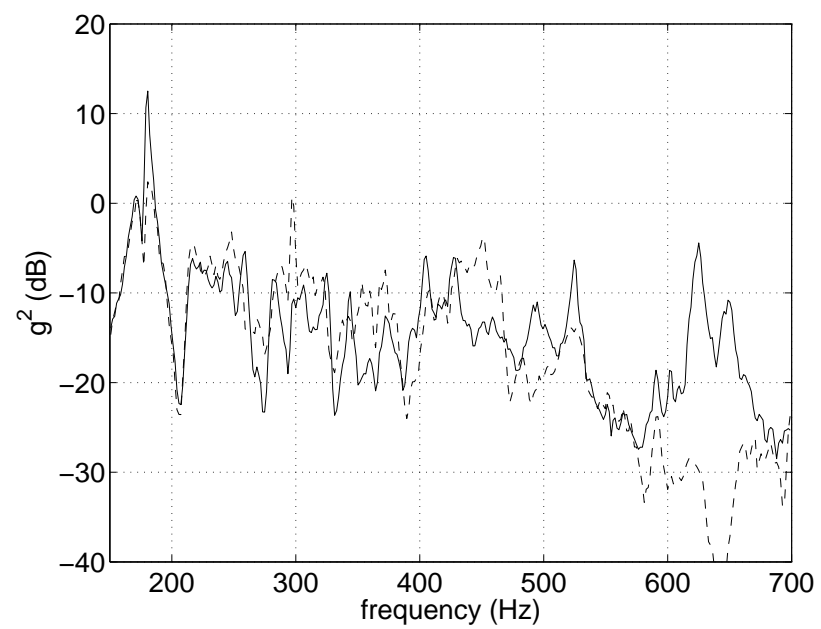

Fig. 10 Auto-spectrum of diamond accelerometer array on bay 1: Open loop (solid line); decentralized control of bays 1 and 2 (dashed line).

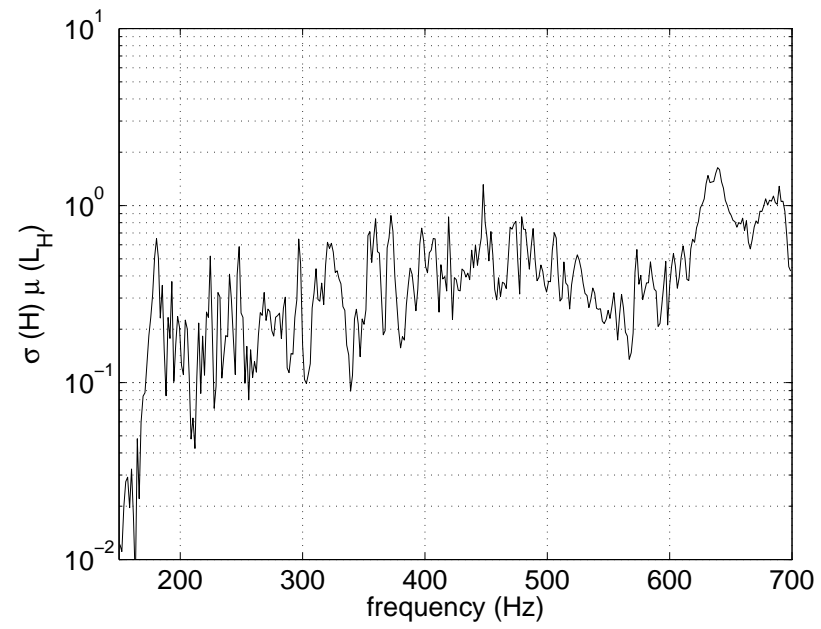

Fig. $11 \bar{\sigma}(\bar{H}) \mu\left(L_{H}\right)$ for decentralized control of bays 1 and 2

constraint in Eq. 14 at most frequencies. Although the constraint violation at $450 \mathrm{~Hz}$ may explain the spillover seen at that frequency, no such spillover occurred at $620 \mathrm{~Hz}$, where the constraint was also violated. Similarly, the constraint in Fig. 11 does not offer insight into the spillover at $300 \mathrm{~Hz}$, since it does not indicate a problem in that area.

Results for the second control configuration are shown in Fig. 12. The figure shows the auto-spectra of the sensor response on bay one for decentralized control of bays 1 and 4 . As with bays one and two, the controller produced good attenuation near $180 \mathrm{~Hz}$ but spilled over near $300 \mathrm{~Hz} \cdot \bar{\sigma}(\bar{H}) \mu\left(L_{H}\right)$ for this case is shown in Fig. 13. This constraint exceeds unity at more frequencies than for bays one and two. There is a peak in Fig. 13 at $300 \mathrm{~Hz}$ which could explain the spillover seen there. However, spillover is not seen in

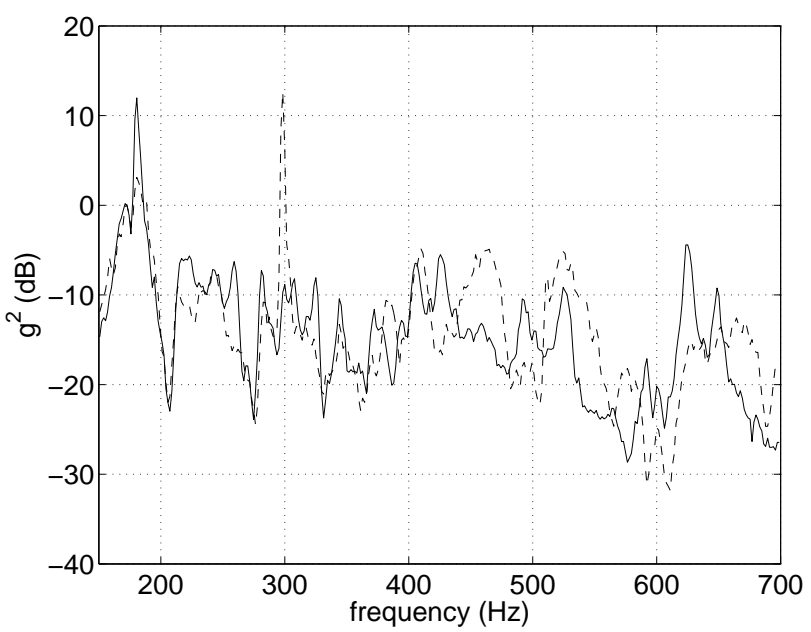

Fig. 12 Auto-spectrum of diamond accelerometer array on bay 1: Open loop (solid line); decentralized control of bays 1 and 4 (dashed line).

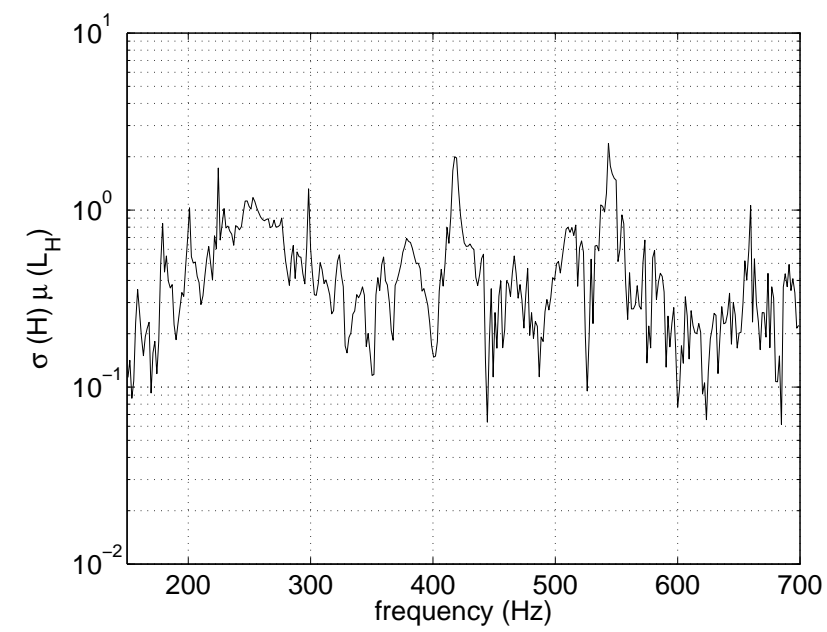

Fig. $13 \bar{\sigma}(H) \mu\left(L_{H}\right)$ for decentralized control of bays 1 and 4

other frequency ranges where the constraint was violated, such as at $225 \mathrm{~Hz}$ or $420 \mathrm{~Hz}$.

\section{Conclusions}

Interaction metrics which were previously described for analyzing decentralized controllers ${ }^{14}$ were applied to experimentally measured data from a model of an aircraft sidewall. Frequency response data from pairs of bays on a fuselage panel were examined. The Relative Gain Array, or RGA, was studied as a means to quantify the diagonal dominance of a plant. The second interaction metric studied was based on a $\mu$ analysis of the off diagonal terms of the plant relative to the diagonal terms. The $\mu$ metric provided a quantitative constraint on the maximum singular value of the complementary sensitivity of a decentralized controller in order to guarantee stability. 
On the panel studied here, the RGA and the $\mu$ metric showed that bays sharing a short vertical boundary were less coupled than bays sharing a long horizontal boundary. Both metrics also indicated that most coupling occurred when the resonance of one bay coupled into the resonance of a neighboring bay, as would be expected. For bays sharing a long horizontal boundary, satisfying the $\mu$ constraint in order to guarantee stability of the decentralized controller could greatly complicate the control design procedure. Further analysis is needed to accurately correlate inter-bay coupling to structural characteristics such as shared boundaries.

Control designs from a previous experiment were found to violate the $\mu$ interaction constraint at several frequency points, although the controllers were stable when implemented. This verifies the conservativeness of this constraint. Spillover produced by the controllers was not easily correlated with violations of the $\mu$ constraint.

More work needs to be done on applying these metrics to this problem. The next step is to use the $\mu$ constraint in the design of decentralized controllers. It will be important to determine if the resulting control designs are too conservative to produce acceptable noise reduction performance.

\section{References}

${ }^{1}$ Gibbs, G., Cabell, R., and Juang, J., "Controller Complexity for Active Control of TBL Induced Sound Radiation from Panels," 6th AIAA/CEAS Aeroacoustics Conference, No. AIAA 2000-2043, Lahaina, Hawaii, June 12-14 2000.

${ }^{2}$ Johnson, M. and Elliott, S., "Active control of sound radiation using volume velocity cancellation," Journal of the Acoustical Society of America, Vol. 98, No. 4, 1995, pp. 2174-2186.

${ }^{3}$ Clark, R. L. and Cox, D. E., "Multi-variable structural acoustic control with static compensation," Journal of the Acoustical Society of America, Vol. 102, No. 5, Nov. 1997, pp. 2747-2756.

${ }^{4}$ Maury, C., Gardonio, P., and Elliott, S. J., "Active Control of the Flow-Induced Noise Transmitted Through a Panel," AIA A Journal, Vol. 39, No. 10, Oct. 2001, pp. 1860-1867.

${ }^{5}$ Baumann, W. T., Saunders, W. R., and Robertshaw, H. H., "Active suppression of acoustic radiation from impulsively excited structures," Journal of the Acoustical Society of America, Vol. 90, No. 6, Dec. 1991, pp. 3202-3208.

${ }^{6}$ Thomas, D. and Nelson, P., "Experiments on the active control of broadband sound radiation from a lightweight partition," Journal of Sound and Vibration, Vol. 202, 1997, pp. 438445.

${ }^{7}$ Yung, J. and Crawley, E., "Compensation Methodologies for Local Control using Strain Actuators and Sensors," SPIE Conference on Smart Structures and Integrated Systems, Newport Beach, March 1999, pp. 913-924.

${ }^{8}$ Gibbs, G. P., Eure, K. W., and Lloyd, J. W., "Active Control of Turbulent Boundary Layer Induced Sound Radiation from Aircraft Style Panels," Proceedings of Active-99, Ft. Lauderdale, Florida, Dec. 1999.

${ }^{9}$ Gibbs, G. P. and Cabell, R. H., "Active Control of Turbulent Boundary Layer Induced Sound Radiation from Multiple
Aircraft Panels," 8th AIAA/CEAS Aeroacoustics Conference, No. AIAA 2002-2496, Breckenridge, CO, June 17-19 2002.

${ }^{10}$ Cox, D., Gibbs, G., Clark, R., and Vipperman, J., "Experimental Robust Control of Structural Acoustic Radiation," ASME Journal of Vibration and Acoustics, Vol. 121, 1999, pp. 433-439.

${ }^{11}$ Skogestad, S. and Postelthwaite, I., Multivariable Feedback Control: Analysis and Design, John Wiley and Sons, 1996.

${ }^{12}$ Elliott, S. J., Gardonio, P., Sors, T. C., and Brennan, M. J., "Active Vibroacoustic Control with Multiple Local Feedback Loops," Journal of the Acoustical Society of America, Vol. 111, No. 2, Feb. 2002, pp. 908-915.

${ }^{13}$ Sun, J., "Some Observations on Physical Duality and Colocation of Structural Control Sensors and Actuators," Journal of Sound and Vibration, Vol. 194, No. 5, 1996, pp. 765-770.

${ }^{14}$ Morari, M. and Zafiriou, E., Robust Process Control, Prentice Hall, Upper Saddle River, New Jersey, 1998.

${ }^{15}$ Anderson, B. and Moore, J., Optimal Control - Linear Quadratic Methods, Information and System Sciences Series, Prentice Hall, Englewood Cliffs, New Jersey, 1990.

${ }^{16}$ Zhou, K., Doyle, J. C., and Glover, K., Robust and Optimal Control, Prentice Hall, 1996.

${ }^{17}$ Packard, A. and Doyle, J., "The Complex Structured Singular Value," Automatica, Vol. 29, No. 1, 1993, pp. 71-109.

${ }^{18}$ Clarke, D., Mohtadi, C., and Tuffs, P., "Generalized Predictive Control - Part I. The Basic Algorithm," Automatica, Vol. 23, No. 2, 1987, pp. 137-148.

${ }^{19}$ Juang, J.-N. and Phan, M., "Deadbeat Predictive Controllers," Tech. Rep. TM-112862, NASA Langley Research Center, May 1997.

${ }^{20}$ Juang, J.-N., Applied System Identification, Prentice Hall, 1994.

10 of 10

American Institute of Aeronautics and Astronautics 